\title{
Brandschutzhelferkurse in München
}

\author{
Der Brandschutz hat jetzt auch einen hohen Stellenwert für die Zahnarztpraxen in Deutschland \\ erhalten. Die Technische Regel für Arbeitsstätten, ASR A2.2 Maßnahmen gegen Brände, fordert \\ von jedem Arbeitgeber (also auch vom Praxisinhaber) die Benennung von Brandschutzhelfern. \\ Die vom Arbeitgeber benannten Personen sind im Notfall dafür zuständig, einen Brand zu be- \\ kämpfen oder eine Evakuierung einzuleiten. Mindestens muss jedoch ein Brandschutzhelfer je \\ Arbeitsstätte vorhanden sein.
}

Dies kann auch die Praxisinhaberin oder der Praxisinhaber sein. Die Ausbildung zum Brandschutzhelfer besteht laut der Berufsgenossenschaft für Gesundheitsdienst und Wohlfahrtspflege (BGW) aus einer theoretischen Unterweisung und einer praktischen Übung. Für die Theorie gibt es zwei Unterrichtseinheiten von je 45 Minuten, in denen zum Beispiel die Zusammenarbeit mit der Feuerwehr, Brandursachen und Brandarten behandelt werden. Die praktischen Übungen sollen jedem die Möglichkeit geben, realitätsnahe Übungen mit Feuerlöscheinrichtungen $\mathrm{zu}$ absolvieren.

In München hat die Bezirksgruppe des Freien Verbandes Deutscher Zahnärzte mehrere Kurse zur Ausbildung zum Brandschutzhelfer mithilfe der bayerischen Zahnärztegenossenschaft ABZeG in deren Räumen und mit deren logistischer Unterstützung bei der Durchführung abgehalten. Das Angebot fiel auf fruchtbaren Boden, da die durch verschiedene Artikel in der Standespresse sensibilisierte Kollegenschaft auf das Angebot wartete. In kurzer Zeit waren alle Kursplätze vergeben, so dass weitere Termine gebucht und natürlich auch gleich wieder ausgebucht waren. Da die Verordnung nicht nur Zahnarztpraxen, sondern alle Arbeitsstätten betrifft, sind Ausbilder und potenzielle Kursanbieter stark ausgelastet. In München ist man in der glücklichen Lage gewesen, dass sich der Kursanbieter auf die Erfordernisse der Zahnärzte eingestellt hat und den bei Zahnärzten oftmals freien Nachmittag des Mittwochs Monate im Voraus für die Kurse blockte und das Kursangebot darstellt.

Bei den Teilnehmern war die anfängliche Skepsis und der Unmut über die neue Auflage schnell geschwunden, denn der launige
Kursleiter verstand es, das Programm auf das für die Zahnarztpraxen Notwendige und im Alltag Wichtige herunterzubrechen und anhand von kleinen Filmchen visuell zu unterlegen.

Die praktischen Übungen trafen dann genau ins Herz der Zahnärzte, die gerne das Theoretische in die Praxis umsetzen möchten. Es hat auch den Damen unter den Teilnehmern Spaß gemacht, mit dem lauten aber effektiven Löschgerät (siehe Bild) umzugehen. Ein runder Vormittag oder Nachmittag war es für die zufriedenen Teilnehmer, als sie das ideale Übungsgebiet im Innenhof des Verwaltungsgebäudes der ABZeG verließen. Die anfängliche Skepsis war vollkommen verschwunden und neue Erkenntnisse und Wissenswertes auch für den Alltag gab es auch noch. Für Ausbildungskurse kann man gerne bei Bezirksgruppen des FVDZ anfragen, ob ein Kurs angeboten wird oder Adressen von Anbietern bekannt sind.

Zusätzlich muss man regelmäßige Unterweisungen des Praxispersonals durchführen, bei denen alle Beschäftigten mindestens einmal jährlich über die in ihrem Arbeitsbereich vorhandenen Brandgefahren und Brandschutzeinrichtungen sowie das Verhalten im Gefahrenfall unterwiesen werden müssen. Dazu bieten sich beispielsweise kontinuierliche Informationen und regelmäßige Informationsveranstaltungen im Rahmen der innerbetrieblichen Kommunikation an. Neue Mitarbeiter sind im Rahmen einer Erstunterweisung über die wichtigsten betrieblichen Brandschutzaspekte zu informieren. Die Unterweisungen sind zu dokumentieren.

Dr. Christian Öttl

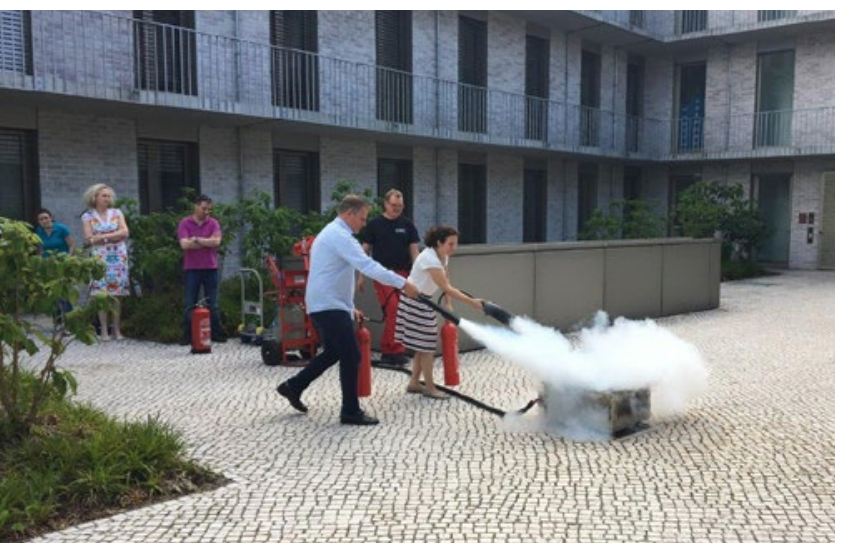

Löschübunngen im Innenhof des Bürogebäudes

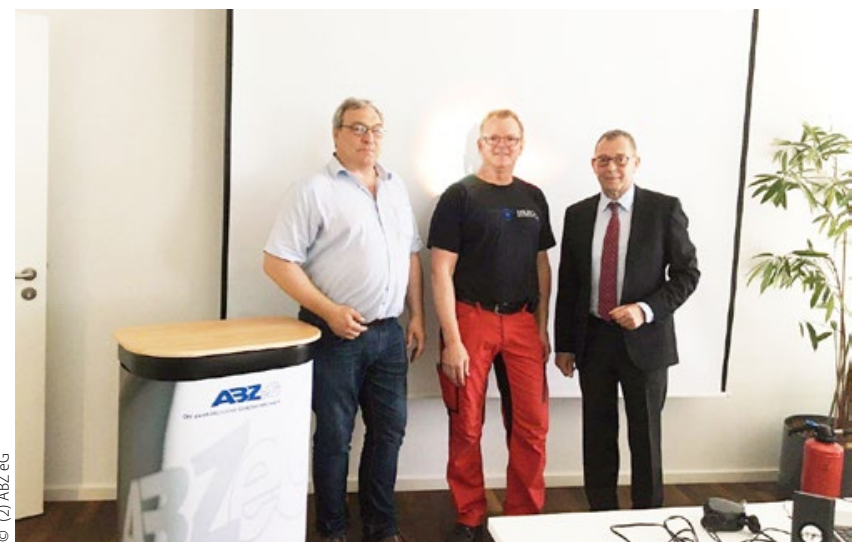

Von rechts: Heinz Abler, Vorstand ABZeG, Roland Falkenstörfer, HERO Brandschutz, Dr. Christian Öttl, FVDZ-Bundesvorstandsmitglied 